\title{
Research on the Existing Problems and Countermeasures of Human Resource Management in Public Institutions
}

\author{
Li Haiping \\ Changqing Yellow River Bureau, Jinan Yellow River Bureau, Jinan, China
}

\section{Email address:}

35195341@qq.com

\section{To cite this article:}

Li Haiping. Research on the Existing Problems and Countermeasures of Human Resource Management in Public Institutions. Science Innovation. Vol. 9, No. 4, 2021, pp. 170-172. doi: 10.11648/j.si.20210904.20

Received: May 20, 2021; Accepted: June 25, 2021; Published: July 9, 2021

\begin{abstract}
Public Institutions need to deal with different management affairs in operation, human resource management is one of the important work. This article takes this as the discussion object, carries on the analysis on how to improve the human resources management quality, simultaneously also has analyzed the question which appears in the human resources management, has given the enhancement human resources management countermeasure, integrating advanced ideas into human resource management, in order to change the staff's traditional management thought, and combine the development strategy of the unit to perfect the ideological connotation, so as to stimulate the staff's enthusiasm for work, at the same time, we will improve the information level of human resources management, continuously optimize the allocation of human resources, strengthen the pertinence and effectiveness of staff training, and provide specialized training combining with the work positions of staff, so as to improve the work level of staff, improve staff's work ability, detailed reward, through the perfect salary management system to mobilize staff work innovation, to ensure that their services to keep up with the times, and ultimately improve the quality of human resources management.
\end{abstract}

Keywords: Public Institutions, Human Resources, Management

\section{事业单位人力资源管理存在的问题及对策研究}

\section{李海平}

长清黄河河务局, 济南黄河河务局, 济南, 中国

\section{邮箱}

35195341@qq.com

摘要: 事业单位运行中需要处理不同管理事务, 人力资源管理属于其中一项重要的工作内容。本文以此为探讨对象, 就如何提高人力资源管理质量进行分析，同时也分析了人力资源管理中出现的问题，给出了提高人力资源管理的对 策, 将先进的理念融入到人力资源管理工作之中, 以转变工作人员的传统管理思想, 结合单位的发展战略完善思想 内涵, 以此激发员工工作积极性, 同时提升人力资源管理的信息化水平，不断优化人力资源配置，加强员工培训的 针对性和有效性, 结合员工的工作岗位提供专项化的培训, 提高员工的工作水平, 提高员工的工作能力, 细化奖励, 通过完善的薪酬管理体制调动员工工作的创新性, 确保自身各项服务跟上时代发展潮流, 最终提高人力资源管理的 质量。

关键词: 事业单位, 人力资源, 管理 


\section{1. 引言}

事业单位本质上是为社会提供公共服务, 事业单位关 系到社会福祉, 同时也关系到国民幸福水平。为此, 人力 资源管理的质量将直接影响到单位的管理效率, 因此事业 单位需要重视管理, 需要提高人力资源管理效率 [1]。规范 事业单位工作人员工作质量, 可以有效激发事业单位为人 民群众服务的热情, 同时也可以为事业单位自身发展创造 良好的环境。探讨全新的人力资源管理方式方法, 人力资 源管理部门可以使用新方法管理人力资源, 同时还需要创 建完善的管理体系, 以此激发员工工作积极性, 根据员工 的能力确定员工的岗位, 从而充分实现人才的价值, 实现 人力资源的合理配置。通过本文研究可以更好的发现事业 单位人力资源管理中出现了漏洞, 同时也可以提升事业单 位人力资源管理的质量, 以提高事业单位人力资源管理效 率, 积累事业单位人力资源管理的相关理论, 提升管理效 率，具有非常重要的实践意义。李炳舒，杨明（2019）指 出, 事业单位人力资源管理内容丰富, 但是招聘是人力资 源管理的基石, 因此事业单位需要加强招聘管理工作, 以 此提高人力资源管理的效率。

刘倩倩 (2020) 指出, 事业单位人力资源管理需要加 强人力资源的激励, 通过良好的激励提高人员的工作效率。

\section{2. 事业单位人力资源管理的概述}

人力资源管理的内涵非常丰富, 不仅包含有人员招聘, 同时还需做好人员管理工作，而这都是切实做好事业单位 人力资源管理的重要工作内容。一般而言, 事业单位人力 资源管理具有以下几方面特点[2]。

第一，复杂性。我国具有众多事业单位，事业单位涵 盖有医疗、教育、建筑等各行业, 而各行业性质并不相同, 不同行业有着不同的管理模式和方法, 人力资源管理的方 法也不尽相同 [3]。例如, 事业单位员工招聘非常灵活, 不 同行业会根据自身行业特点招聘, 不同人员都是在培训方 面侧重点也不相同, 这会导致事业单位人力资源管理非常 复杂。

第二，服务性。在事业单位内部，人力资源管理工作 主要是为了提升事业单位公共服务质量, 因此事业单位人 力资源管理突出服务性质特点, 以提升为人民服务质量作 为人力资源管理落脚点 $[4]$ 。

第三, 规范性。事业单位都属于正规单位, 对各项工 作都有严格要求, 对人力资源管理工作具有一系列规定, 从人员招聘到最后人员离职都有系统性规定, 因此事业单 位人力资源管理具有规范性特点 $[5,6]$ 。

\section{3. 事业单位人力资源管理存在的问题}

第一，管理理念思想陈旧。目前，事业单位人力资源 管理部门的思想并不先进, 很多工作人员的思想依旧停留 在数十年前, 管理思想没有跟上时代发展潮流, 影响事业 单位人力资源管理质量。由于事业单位缺乏现代化管理理 念, 最终会影响到事业单位人力资源管理质量, 影响单位
管理水平, 影响服务质量, 也没有真正发挥事业单位工作 人员的工作潜力 [7]。例如，一些管理人员的思想陈旧，在 人力资源管理时依旧采用的是论资排辈的思想, 并没有树 立起按照工作能力表现确定岗位。

第二，用人机制不完善。目前，事业单位在用人机制 方面存在一些漏洞, 在人才选拔时缺乏必要的透明度, 甚 至可能会存在暗箱操作的现象 $[8]$ 。近些年来, 虽然事业单 位在不断改善选人用人机制, 但是由于遗留问题较为复杂, 无法在短期之内完全解决这些问题, 致使一些负面因素依 旧存在, 影响事业单位选人用人机制, 不利于优秀人才脱 颖而出。例如, 一些事业单位没有制定完善的人员选拔机 制, 人员选拔完全是根据入职年限的长短确定。

第三，奖惩机制不完善。完善的奖惩制度可以提升事 业单位工作人员工作积极性, 可以规范事业单位工作人员 工作质量, 可以有效激发事业单位为人民群众服务的热情, 同时也可以为事业单位自身发展创造良好的环境[9]。但是， 一些事业单位并没有建立起完善的奖惩制度, 很可能会导 致员工工作积极性不够, 同时也会面临着人才流失局面, 致使事业单位在发展中遇到各种各样的困境。在人才招聘 过程之中, 由于缺乏完善的奖惩机制, 一些高素质人才并 不愿意加入事业单位, 更愿意进入一些奖惩措施更加完善 的单位。除此之外, 现有事业单位奖惩机制单一, 奖惩标 准也单一化, 这些都会影响到事业单位员工工作积极性, 容易导致事业单位员工出现大锅饭的思想, 影响事业单位 自身的发展。

第四, 配置不科学。事业单位人力资源配置不够高效, 人力资源管理不精简, 存在严重的兄员现象, 同时事业单 位也缺乏健全的培训和淘汰制度, 致使事业单位人力资源 管理效率低下, 用人成本较高 $[10]$ 。与此同时, 由于人力 资源配置不合理, 致使一些有才干的员工离开了事业单位, 致使事业单位人才流失。例如, 一些单位在配置人员岗位 时并没有根据人岗匹配的思想配置不科学, 专业不对口。

第五, 缺乏完善的培训制度。目前, 事业单位的培训 制度并不完善, 许多事业单位甚至没有培训制度培训工作, 只是借鉴其他单位的培训工作, 没有结合单位的具体状况, 同时也没有结合人力资源管理的各项工作开展培训, 致使 培训工作千篇一律, 影响员工的工作技能, 同时也影响人 力资源管理的水平 $[11]$ 。与此同时, 在完成培训之后, 事 业单位也没有对员工的培训状况进行分析和调查, 导致培 训工作走过场无法提高员工的工作技能。

\section{4. 事业单位人力资源管理对策}

第一, 创新理念。为提升人力资源管理水平, 事业单 位首先需要学习先进的理念, 将先进的理念融入到人力资 源管理工作之中, 以转变工作人员的传统管理思想, 同时 还需要结合单位的发展战略完善思想内涵, 确保创新思想 可以与企业发展战略结合在一起, 以新的思想指导人力资 源管理工作, 以此提升员工工作的积极性, 从而推动事业 单位人力资源管理工作上一个新台阶[12]。

第二, 创新管理模式。为了有效提升管理水平, 事业 单位需要不断创新管理模式, 探讨全新的人力资源管理方 
式方法, 人力资源管理部门可以使用新方法管理人力资源, 同时还需要创建完善的管理体系, 以此激发员工工作积极 性, 同时提升人力资源管理的信息化水平, 最终提高人力 资源管理的质量。例如, 事业单位人力资源管理部门可以 创新员工激励措施，同时还可以设置员工职业生涯发展规 划, 以此增强员工对事业单位认同感, 使员工更好的为事 业单位服务。

第三, 细化各项奖惩措施。为提升管理质量, 事业单 位需要加强薪酬管理, 提高薪酬管理的公平性和公正性, 以此提升员工的工作效率, 提升事业单位的管理质量。为 此, 事业单位需要结合事业单位自身特点, 不断细化各项 管理流程, 同时还需要完善薪酬管理体制, 通过完善的薪 酬管理体制调动员工工作的创新性, 确保自身各项服务跟 上时代发展潮流[13]。与此同时, 事业单位还需要出台完 善的考核机制，通过完善的考核机制激发员工工作的积极 性, 以此提升员工工作的质量。

第四, 加强培训。在人力资源管理中, 培训也是一项 重要的管理内容, 加强培训是提升管理水平的重要方式, 加强培训可以提高员工的工作水平, 以此提升人力资源管 理质量。为此, 事业单位需要丰富员工培训内容, 需要加 强员工培训的针对性和有效性, 同时还需要结合员工的工 作岗位提供专项化的培训, 提高员工的工作水平, 提高员 工的工作能力, 提升人力资源管理质量[14]。例如, 事业 单位可以每年利用业务淡季开展人员培训工作, 在人员培 训之前加强培训调查, 以此提升培训的专业性和针对性。

第五, 优化人力资源配置。为提升事业单位人力资源 管理质量, 事业单位还需要不断优化人力资源配置, 需要 打破传统的论资排辈的模式, 根据员工的能力确定员工的 岗位, 从而充分实现人才的价值, 实现人力资源的合理配 置。为此, 事业单位可以以 2 年为一个周期, 通过 2 年观察 员工在岗位上的工作表现, 以此确定员工是否胜任工作岗 位, 如果发现不胜任者需要及时淘汰以此, 优化人力资源 管理水平，增强事业单位服务质量[15]。

\section{5. 结语}

综上所述, 为提升人力资源管理质量, 事业单位需转 变管理思想, 探讨全新的人力资源管理方式方法, 人力资 源管理部门可以使用新方法管理人力资源, 创新管理理念, 将先进的理念融入到人力资源管理工作之中, 转变工作人 员的传统管理思想, 创新管理模式, 创建完善的管理体系, 以此激发员工工作积极性, 同时提升人力资源管理的信息 化水平, 细化奖励, 通过完善的薪酬管理体制调动员工工 作的创新性, 确保自身各项服务跟上时代发展潮流, 同时
还需要加强人员培训与管理工作, 结合员工的工作岗位提 供专项化的培训, 提高员工的工作水平, 提高员工的工作 能力, 以此提高人力资源管理水平, 发挥人力资源的优势。

\section{参考文献}

[1] 李炳舒, 杨明. 我国事业单位人力资源管理现状的分析 [J]. 环球市场, 2019,000(005):109-109.

[2] 刘国梁. 论事业单位的人力资源管理与绩效考核 [J]. 财经 界学术版, 2015.

[3] 刘倩倩. 事业单位人力资源管理中的激励机制[J]. 时代金 融, 2020(30):228-228.

[4] 李芗. 关于如何增强事业单位人力资源管理能力问题的思 考 [J]. 商情, 2019, 000(037):137.

[5] 潘静. 利用信息技术进行事业单位人力资源管理的可行性 [J]. 经营管理者, 2014.

[6] 孙刚超. 事业单位人力资源管理及开发分析 [J]. 经贸实践, 2017.

[7] 田园. 事业单位人力资源管理存在的问题及改革措施 [J]. 人民论坛中旬刊, 2013.

[8] 杨秀岩. 事业单位人事管理中的激励机制探索 [J]. 商情, 2019, 000(050):130.

[9] 杨迪. 事业单位财会人力资源管理存在问题及对策研究 [J]. 财会学习, 2020(27).

[10] 王文婷. 优化人力资源配置 提升事业单位经济效益 $[\mathrm{J}]$. 中 外企业家,2016.

[11] 姜友文. 欠发达地区高职院校人力资源管理存在的问题及 对策研究[J].学问, 2009.

[12] 张车伟. 中国人口年鉴2007[J]. 中国人口年鉴杂志社, 2007.

[13] 贾智博. 事业单位人力资源管理在变革中的问题及建议 [J]. 中国商界上半月, 2012 .

[14] 冯春芳. 论事业单位人力资源管理 [J]. 现代经济信息, 2015.

[15] 李勃. 关于创新事业单位人力资源管理的探讨 [J].城市建设 理论研究, 2019. 